INPLASY

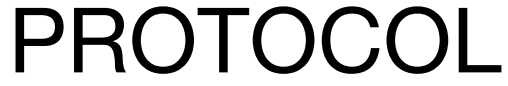

To cite: Dioguardi et al. The

Prognostic Role of miR-31 and miR-155 in HNSCC: Protocol. Inplasy protocol 202210119. doi:

10.37766/inplasy2022.1.0119

Received: 25 January 2022

Published: 25 January 2022

Corresponding author:

Mario Dioguardi

mario.dioguardi@unifg.it

Author Affiliation:

University of Foggia.

Support: University of Foggia.

Review Stage at time of this submission: Formal screening of search results against eligibility criteria.

Conflicts of interest:

None declared.

\section{The Prognostic Role of miR-31 and miR-155 in HNSCC: Protocol}

Dioguardi, M1; Sovereto, D2; Troiano, G³.

Review question / Objective: the PICO question was formulated, in which the following points are identified: Participants (patients with HNSCC), Intervention (altered expression of miR-31 and miR-155 in HNSCC), Control (patients with HNSCC who have low expression of miR-31 and miR-155), Outcome (difference in prognosis of survival among patients with low and high miR-31 and miR-155 expression in HNSCC The PICO question therefore is as follows: Is there a difference in OS (overall Survival) between HNSCC patients with high miR-31 and miR-155 expression versus those with low expression?

Condition being studied: Head and Neck Squamous Cell Carcinoma (HNSCC), altered expression of miR-31 and miR-155 tissue.

INPLASY registration number: This protocol was registered with the International Platform of Registered Systematic Review and Meta-Analysis Protocols (INPLASY) on 25 January 2022 and was last updated on 25 January 2022 (registration number INPLASY202210119).

\section{INTRODUCTION}

Review question / Objective: The PICO question was formulated, in which the following points are identified: Participants (patients with HNSCC), Intervention (altered expression of $\mathrm{miR}-31$ and $\mathrm{miR}-155$ in
HNSCC), Control (patients with HNSCC who have low expression of miR-31 and miR-155), Outcome (difference in prognosis of survival among patients with low and high miR-31 and miR-155 expression in HNSCC The PICO question therefore is as follows: Is there a difference in OS (overall 
Survival) between HNSCC patients with high miR-31 and miR-155 expression versus those with low expression?

Rationale: Over the past 10 years, many studies have investigated the altered expression of miR-31 and miR-155 correlating it with the prognosis and progression of malignancies. A clinical study of 56 patients by Qiang et al. (2019) investigated prognostic potential by identifying a longer survival period in patients in whom miR-31 was less expressed, indicating how this non-coding RNA sequence can be considered a crucial benchmark for the prognosis of patients with HNSCC. , these data are partially in agreement with the results of $\mathrm{Tu}$ et al. (2021) which identifies worse overall survival in patients with higher miR-31 expression. These 2 very recent studies show that miR-31 and miR-155, I can represent a prognostic biomarker of survival; Furthermore, no other systematic reviews were conducted on the prognostic role of the altered expression of miR-31 and miR-155. With this systematic review, we therefore set ourselves the goal of providing a combined HR value (high and low expression of miR- 31 and miR155 on the overall survival (OS) of patients with HNSCC.

Condition being studied: Head and Neck Squamous Cell Carcinoma (HNSCC), altered expression of miR-31 and miR-155 tissue.

\section{METHODS}

Search strategy: Terms: miR-31 AND HNSCC, Microrna AND HNSCC AND prognosis, miR-31 AND OSCC, miR-31 AND laryngeal cancer, miR-31 AND oropharynx, miR-155 AND HNSCC, miR-155 AND OSCC,miR-155 AND laryngeal cancer, miR-155 AND oropharynx databases: Scopus, PubMed, Cochrane Central Register of Controlled Trial, Opengray, Google scholar.

Participant or population: Patients with HNSCC.
Intervention: Altered expression of miR-31 and miR-155 in HNSCC tissue.

Comparator: Overall survival hazard ratio between low and high tissue expression of miR.31 and miR-155 in patients with HNSCC.

Study designs to be included: Retrospective studies, observational studies and RTC.

Eligibility criteria: All retrospective, prospective and randomized trials that considered differences in miR-31and miR-155 expression in patients with HNSCC in correlation with prognostic indices of survival and specific OS were taken into consideration.

Information sources: Scopus, PubMed, Cochrane Central Register of Controlled Trial, Opengray, Google scholar and previous systematic reviews on microRNA.

Main outcome(s): Difference in prognosis of survival among patients with low and high miR-31 and miR-155 expression in HNSCC. Survival HR data will be extracted from the individual reports and aggregated using Revman 5.4 software. The value resulting from the meta-analysis will be expressed as HR Pooled, and will be represented graphically through a forest plot.

Data management: The data will be extracted from 2 reviewers and reported on 2 different tables and subsequently compared to minimize the risk of error in reporting. Subsequently the HR values will be implemented on meta-analysis programs (RevMan, open metanalisis) to obtain an aggregate value of HR with the relative $p$ values and heterogeneity indices.

Quality assessment / Risk of bias analysis: The risk of bias will be assessed through the REMARK scale and the quality of the evidence will be assessed through IL GRADEpro.

Strategy of data synthesis: The data will be summarized and represented through the use of tables where the main 
characteristics of the individual studies included and of the relative patient cohorts will be reported. Furthermore, the data on the expression of miR-31 and miR-155 with the relative HRs on the prognostic indices of survival will be reported. The aggregated data of the HR values will be represented by Forest plot.

Subgroup analysis: With an appropriate number of studies included, a subgroup analysis by HNSCC location could be conducted (OSCC, LSCC; HSCC).

Sensitivity analysis: A sensitivity analysis can be conducted to search for sources of heterogeneity through the graphical analysis of confidence intervals (Forest Plot) through the Funnel plot, if the Higgs heterogeneity index is greater than $50 \%$.

Language: Only clinical studies in English.

Country(ies) involved: Italy.

Keywords: miR-31, miR-155, HNSCC, OSCC, microRNA.

Contributions of each author:

Author 1 - Mario Dioguardi.

Email: mario.diguardi@unifg.it

Author 2 - Diego Sovereto.

Email: diego_sovereto.546709@unifg.it

Author 3 - Giuseppe Troiano.

Email: giuseppe.troiano@unifg.it 